Case Reports in
Gastroenterology
Case Rep Gastroenterol 2020;14:658-667

DOI: $10.1159 / 000511034$

Published online: December 10, 2020
(C) 2020 The Author(s)

Published by S. Karger AG, Basel www.karger.com/crg

This article is licensed under the Creative Commons Attribution-NonCommercial 4.0 International License (CC BY-NC) (http://www.karger.com/Services/OpenAccessLicense). Usage and distribution for commercial purposes requires written permission.

\title{
Prolonged Survival of a Patient with Advanced-Stage Combined Hepatocellular-Cholangiocarcinoma
}

\author{
Sven H. Loosen ${ }^{a, b} \quad$ Nadine T. Gaisa ${ }^{c} \quad$ Maximilian Schmeding ${ }^{d}$ \\ Christoph Heining ${ }^{e, f}, g$ Sebastian Uhrigh, ${ }^{h}$ Theresa H. Wirtz ${ }^{b}$ \\ Sebastian Kalverkamp ${ }^{j}$ Jan Spillner ${ }^{j} \quad$ Frank Tacke $^{k}$ \\ Albrecht Stenzinger ${ }^{l, m} \quad$ Hanno Glimm ${ }^{e, f, g, n} \quad$ Stefan Fröhling ${ }^{m, o}$ \\ Christian Trautwein $^{b} \quad$ Christoph Roderburg $^{a} \quad$ Thomas Longerich $^{p}$ \\ Ulf Peter Neumann ${ }^{q, r}$ Tom Luedde ${ }^{a, b}$
}

${ }^{a}$ Clinic for Gastroenterology, Hepatology and Infectious Diseases, University Hospital Düsseldorf, Medical Faculty of Heinrich Heine University Düsseldorf, Düsseldorf, Germany; ${ }^{b}$ Department of Medicine III, University Hospital RWTH Aachen, Aachen, Germany; 'Institute of Pathology, University Hospital RWTH Aachen, Aachen, Germany; ${ }^{\text {dDepartment }}$

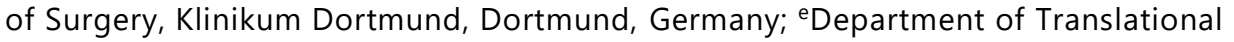
Medical Oncology, National Center for Tumor Diseases (NCT) Dresden, Dresden, Germany; ${ }^{f}$ Center for Personalized Oncology, NCT Dresden and University Hospital Carl Gustav Carus Dresden, Technical University Dresden, Dresden, Germany; gGerman Cancer Consortium (DKTK), Dresden, Germany; hDivision of Applied Bioinformatics, DKFZ, Heidelberg, Germany; 'Molecular Diagnostics Program, NCT Heidelberg and DKFZ, Heidelberg, Germany; jDepartment of Thoracic and Cardiovascular Surgery, University Hospital RWTH Aachen, Aachen, Germany; ${ }^{k}$ Department of Gastroenterology/Hepatology, Campus Virchow Klinikum and Campus Charite Mitte, Charité - Universitätsmedizin Berlin, Berlin, Germany; 'Institute of Pathology, Heidelberg University Hospital, Heidelberg, Germany; ${ }^{m}$ German Cancer Consortium (DKTK), Heidelberg, Germany; ${ }^{n}$ Translational Functional Cancer Genomics Group, NCT Heidelberg and DKFZ, Heidelberg, Germany; ${ }^{\circ}$ Department of Translational Medical Oncology, NCT Heidelberg and DKFZ, Heidelberg, Germany; PInstitute of Pathology, University Hospital Heidelberg, Heidelberg, Germany; "Department of Visceral and Transplantation Surgery, University Hospital RWTH Aachen, Aachen, Germany; 'Department of Surgery, Maastricht University Medical Centre (MUMC), Maastricht, The Netherlands 


\section{Case Reports in Gastroenterology}

\begin{tabular}{l|l}
\hline Case Rep Gastroenterol 2020;14:658-667 \\
\hline DOI: 10.1159/000511034 & $\begin{array}{l}\text { ○ 2020 The Author(s). Published by S. Karger AG, Basel } \\
\text { www.karger.com/crg }\end{array}$ \\
\hline
\end{tabular}

Loosen et al.: Interdisciplinary Therapeutic Approach to Advanced-Stage cHCC/CCA

\title{
Keywords
}

Combined hepatocellular-cholangiocarcinoma - Liver transplantation · Chemotherapy . Next-generation sequencing · Targeted therapy

\begin{abstract}
Combined hepatocellular-cholangiocarcinoma (cHCC/CCA) represents a rare type of primary liver cancer with a very limited prognosis. Although just recently genomic studies have contributed to a better understanding of the disease's genetic landscape, therapeutic options, especially for advanced-stage patients, are limited and often experimental, as no standardized treatment protocols have been established to date. Here, we report the case of a 38-year-old male patient who was diagnosed with extensive intrahepatic cHCC/CCA in an otherwise healthy liver without signs of chronic liver disease. An interdisciplinary stepwise therapeutic approach including locoregional liver-targeted therapy, systemic chemotherapy, liver transplantation, surgical pulmonary metastasis resection, and next-generation sequencing-based targeted therapy led to a prolonged overall survival beyond 5 years with an excellent quality of life. This case report comprises several provocative treatment decisions that are extensively discussed in light of the existing literature on this rare but highly aggressive malignancy.
\end{abstract}

(C) 2020 The Author(s)

Published by S. Karger AG, Basel

\section{Introduction}

Combined hepatocellular-cholangiocarcinoma (cHCC/CCA) represents a rare but highly aggressive primary liver cancer featuring disease characteristics of both hepatocellular carcinoma (HCC) and cholangiocarcinoma (CCA). Incidence rates are around 0.5 per 1,000,000 people and year and have been increasing over the last decades [1]. The overall prognosis of patients with cHCC/CCA has remained poor, with a median overall survival of just 9 months for all tumor stages combined [1]. Surgical resection represents the only curative therapy, while liver transplantation (LT), which is mainly conducted due to initial misdiagnosis, has shown no clinical benefit over tumor resection [2]. For unresectable cases, nonsurgical treatment options include transarterial chemoembolization (TACE), radioembolization, or systemic chemotherapy, but reliable data on the efficacy especially of liver-directed therapies are missing [3]. With respect to systemic treatment, smaller series have revealed a benefit of platinum-based chemotherapy (e.g., gemcitabine/cisplatin) over tyrosine kinase inhibitors (e.g., sorafenib) [3], but no randomized controlled trials are available. As disease-specific therapeutic options are missing and standardized treatment protocols do not exist, cHCC/CCA patients often undergo an individual and partly experimental therapeutic approach with divergent clinical success.

Here, we report the case of a young patient with advanced-stage cHCC/CCA in which an interdisciplinary personalized therapeutic approach that included a stepwise sequence of locoregional therapy, systemic chemotherapy, LT, surgical resection, and molecularly targeted therapy led to a prolonged overall survival beyond 5 years.

\section{Karger'"}




\section{Case Reports in Gastroenterology}

Case Rep Gastroenterol 2020;14:658-667 DOI: 10.1159/000511034

Loosen et al.: Interdisciplinary Therapeutic Approach to Advanced-Stage cHCC/CCA

\section{Case Report}

A 38-year-old male Caucasian patient was referred to the outpatient clinic in April 2014 with unexplained weight loss ( $4 \mathrm{~kg}$ in 6 months), physical exhaustion, and fatigue, as well as elevated liver enzymes. The patient was an otherwise healthy man (ECOG PS 0) who, despite mild obesity (BMI $28.4 \mathrm{~kg} / \mathrm{m}^{2}$ ), had no relevant medical history. A CT scan revealed multiple hypodense liver lesions in all liver segments with a maximum tumor diameter of $12.9 \mathrm{~cm}$, as well as two suspicious hepatic lymph nodes, but no sign of distant metastases (Fig. 1a). The tumor revealed a slight peripheral hyperenhancement in the venous phase.

A PET-CT scan confirmed the presence of liver-limited disease with multiple intrahepatic sites of FDG uptake but no signs of hypermetabolism in the suspicious hepatic lymph nodes (Fig. 1b). The patient's tumor marker levels (alpha-fetoprotein [AFP]: 1,031.0 ng/mL; carcinoembryonic antigen: $89.1 \mathrm{ng} / \mathrm{mL}$; CA19-9: 4,516 U/mL) and the unspecific contrast agent uptake were suggestive of $\mathrm{cHCC} / \mathrm{CCA}$. Other routine laboratory markers of liver, kidney and bone marrow function were within the normal range. A CT-guided liver biopsy revealed a poorly differentiated keratin 7-positive adenocarcinoma, which was morphologically most consistent with a CCA (Fig. 2a). Although AFP expression could not be demonstrated in the tumor tissue (Fig. 2a), the fact that the serum AFP levels were $>1,000 \mathrm{ng} / \mathrm{mL}$ suggested a cHCC/CCA.

The case was extensively discussed on the interdisciplinary tumor board. Based on promising response rates of $\mathrm{cHCC} / \mathrm{CCA}$ to TACE therapy and given the fact that the tumor burden was limited to the liver (PET-negative, enlarged hepatic lymph nodes) but surgical resectability was not given, we decided to initially treat the patient with bilobar triple-drug TACE (cisplatin, mitomycin, and doxorubicin). Two weeks after TACE, we observed a divergent tumor marker response with decreasing serum AFP levels but increasing CA19-9 levels (Fig. 1c), implying that the HCC component of the suspected combined tumor was responding to the TACE treatment but the CCA component did not.

We thus decided to initiate systemic treatment for advanced-stage CCA. Although the combination of gemcitabine and cisplatin was considered standard of care, promising data from smaller sets of patients, as well as an undergoing phase II clinical trial (PICCA study) evaluating the potential benefit of epidermal growth factor receptor (EGFR) targeting in KRAS wild-type CCA patients [4], led to the decision for a triple therapy with gemcitabine, cisplatin, and panitumumab after confirmation of KRAS wild-type status in our patient. Despite a papulopustular skin rash, which is commonly observed in patients receiving anti-EGFR therapy and in our case was successfully treated with topical phytomenadione (vitamin $\mathrm{K}_{1}$ ) and oral tetracycline (minocycline $50 \mathrm{mg}$ twice daily), the triple systemic therapy was initially well tolerated and resulted in a significant decrease in both AFP and CA19-9 serum levels (Fig. 1c).

The treatment response to the combination of TACE and systemic treatment was further confirmed by a CT scan 5 months after initial diagnosis, which revealed a partial tumor response of almost all hepatic tumor manifestations and no signs of new abdominal or thoracic tumor manifestations (Fig. 1d). However, surgical resectability was still not given, and the patient's bone marrow function was steadily deteriorating, with progressive thrombocytopenia and neutropenia of CTCAE grade 3 and 4 during the last 4 weeks of treatment, urging us to explore further treatment alternatives.

Following extensive discussion on the interdisciplinary tumor board (see Discussion section for details), we decided to consider LT if an extended-criteria allocation offer that would otherwise be discarded became available. After intraoperative exclusion of extrahepatic

\section{Karger'=}




\section{Case Reports in Gastroenterology}

Case Rep Gastroenterol 2020;14:658-667 DOI: $10.1159 / 000511034$

(c) 2020 The Author(s). Published by S. Karger AG, Basel www.karger.com/crg

Loosen et al.: Interdisciplinary Therapeutic Approach to Advanced-Stage cHCC/CCA

tumor manifestations in the two suspicious but PET-negative periportal lymph nodes, the patient underwent LT using an extended-criteria allocation organ that was rejected by several transplantation centers and was unsuitable for all other local transplantation candidates in October 2014. The histology of the explant liver revealed an extensively necrotic adenocarcinoma with cholangiocellular differentiation and regressive changes measuring a maximum diameter of $9 \mathrm{~cm}$ with no signs of lymphovascular invasion (pT2, pN0 (0/3), L0, V0, R0). The patient recovered fast following LT and was then treated with adjuvant gemcitabine and cisplatin for 3 months and received a dual immunosuppressive therapy with tacrolimus $(2 \mathrm{mg}$ once daily) and everolimus (1.25 mg twice daily). The subsequent follow-up examinations (tumor marker assessment and CT and MRI scans) showed no sign of tumor recurrence for 16 months after LT.

In March 2016, however, the patient presented with newly developed pulmonary lesions that were histologically confirmed as metastases of the CCA component of the cHCC/CCA (K19 positive, AFP negative; Fig. 2b). Based on the good initial tumor response to systemic chemotherapy and the recovery of the patient's bone marrow function, we reinduced systemic treatment with gemcitabine and cisplatin, which first resulted in a partial response (after 3 months) and subsequently also in stable disease of all pulmonary lesions.

However, about 15 months after reinduction of systemic therapy (in June 2017), the side effects of chemotherapy worsened again (thrombocytopenia and neutropenia grade 3-4 despite bone marrow stimulation), which made it clinically impossible to continue with this therapy regimen. Given the good pulmonary response to chemotherapy, the absence of additional abdominal or thoracic tumor manifestations, and the inability to continue chemotherapy, we performed a two-stage surgical metastasectomy in June/August 2017 using a "personalized therapeutic approach," from which the patient recovered well.

To identify therapeutically tractable molecular lesions, the patient was enrolled in the MASTER (Molecularly Aided Stratification for Tumor Eradication Research) program of the German Cancer Consortium, a registry trial for genomics-guided stratification of younger adults with advanced-stage cancer across all histologies. Whole-genome and RNA sequencing of the resected lung metastases revealed a COLGB1-MET fusion, as well as high FGFR4 and low CDKN2A expression. Based on the promising antitumoral activity of crizotinib - a multityrosine kinase inhibitor that targets ROS, ALK and MET tyrosine kinases - in MET fusionbearing pediatric glioblastoma patients [5], we started systemic therapy with crizotinib when the patient showed recurrence of pulmonary metastases in January 2018. Despite missing data on the usage of crizotinib in liver-transplanted patients receiving immunosuppressive therapy, the drug was well tolerated without clinically significant side effects. The patient showed a partial response/stable disease of almost all pulmonary metastases (after 3 and 7 months, respectively), but we observed a single lesion in the right lower lobe which was increasing in size after 7 months. While continuing treatment with crizotinib, we subsequently performed a video-assisted thoracoscopic resection of this individual metastasis and conducted a second NGS of this metastasis to unravel further genetic alterations that were not sufficiently targeted by crizotinib.

Interestingly, besides the previously observed SMARCA4 deletion, this analysis revealed a high-level FGFR3 gene amplification in the single progressive metastasis. Given that lenvatinib represents a multikinase inhibitor targeting the FGFR family among others, we decided to switch systemic treatment to lenvatinib when the patient showed disease progression in terms of novel bone metastases (humerus, sternum, and lumbar vertebral body 5), as

\section{Karger'=}




\section{Case Reports in Gastroenterology}

Case Rep Gastroenterol 2020;14:658-667 DOI: $10.1159 / 000511034$

(c) 2020 The Author(s). Published by S. Karger AG, Basel www.karger.com/crg

Loosen et al.: Interdisciplinary Therapeutic Approach to Advanced-Stage cHCC/CCA

well as cerebral metastasis, in December 2018. The cerebral and bone metastases were further treated with radiation therapy.

Although the treatment with lenvatinib led to a partial response of the cerebral metastasis and stable disease of the pulmonary metastases at 3 months, we observed progressive bone metastases (lumbar vertebral body 3, 4, and 5) in February 2019. Immunohistochemistry showed a partially necrotic keratin 7-positive adenocarcinoma now also being positive for AFP (Fig. 2c). In a third NGS approach, we observed an activating MET mutation (p.Tyr1230Cys) which has been described as a secondarily acquired resistance mutation following crizotinib treatment, but no other potentially druggable target. Furthermore, the progressive clinical deterioration due to rapid tumor progression made further antitumoral therapy impossible, and we assigned the patient to active symptom control. The patient deceased in June 2019, having survived for 62 months following the diagnosis of advanced-stage cHCC/CCA.

\section{Discussion}

cHCC/CCA is a rare type of liver cancer with a dismal prognosis. Due to its comparatively low incidence rate, the evolving pathophysiological and molecular understanding of the disease, and the high percentage of misdiagnosed patients who are treated either as HCC cases or as CCA cases, no tumor-specific treatment options have been established yet and standardized treatment protocols for $\mathrm{CHCC} / \mathrm{CCA}$ are missing. In this report, we presented the case of a young patient who was diagnosed with advanced-stage cHCC/CCA and who responded exceptionally well to an unconventional personalized therapeutic approach including locoregional liver-directed therapy, systemic chemotherapy, LT, surgical metastasis resection, and precision oncology. Despite the limited availability of data on the efficacy and safety profiles of some of the therapeutic decisions in the context of cHCC/CCA, and especially of their combined application (e.g., NGS-based targeted therapy following LT), the patient showed an unexpected long-term survival beyond 5 years.

As a first line of treatment, we decided on a combination of bilobar triple TACE and systemic chemotherapy with gemcitabine, cisplatin, and panitumumab. The role of locoregional liver-directed therapies for cHCC/CCA has only been poorly understood to date. In 2010, Kim et al. [6] reported a tumor response rate of $70 \%$ following TACE therapy in a series of 50 cHCC/CCA patients, prompting us to choose TACE therapy as a first-line approach for our patient with unresectable advanced but liver-limited tumor manifestation. More recently, it has been shown that combined tumors with a global contrast enhancement pattern benefit to a larger extent from TACE therapy in terms of tumor response and overall patient survival when compared to peripherally enhancing tumors, implying that HCC-dominant cHCC/CCA tumors represent the better TACE candidates [7].

The divergent tumor marker response with increasing levels of CA19-9 three weeks after TACE therapy suggested that the CCA component of the combined tumor had not been treated adequately and prompted the decision for systemic treatment. Although prospective randomized controlled studies are missing, most retrospective meta-analyses have favored CCA-directed systemic treatment (e.g., gemcitabine/cisplatin) over sorafenib for advanced-stage cHCC/CCA [3]. Based on the promising results from a phase II study showing an encouraging antitumor activity with good tolerability of cetuximab in combination with gemcitabine/oxaliplatin [4], as well as from the - at this time - ongoing PICCA trial evaluating the addition of

\section{Karger'=}




\section{Case Reports in Gastroenterology}

Case Rep Gastroenterol 2020;14:658-667

DOI: $10.1159 / 000511034$

c) 2020 The Author(s). Published by S. Karger AG, Basel www.karger.com/crg

Loosen et al.: Interdisciplinary Therapeutic Approach to Advanced-Stage cHCC/CCA

panitumumab to gemcitabine/cisplatin in cases of KRAS wild-type biliary tract cancer, we decided on a triple-drug therapy with gemcitabine, cisplatin, and panitumumab. Although this therapy was well tolerated and revealed an excellent tumor response in our patient, the negative final results from the PICCA trial argue against a benefit from anti-EGFR targeting in CCA. As checkpoint inhibitors (e.g., anti-PD1, anti-PD-L1, and anti-CTLA4) and their combination with other antitumoral drugs are being clinically tested for CCA and have already shown a significant survival benefit in HCC (e.g., IMbrave-150 trial), a potential future application of immunotherapy to $\mathrm{cHCC} / \mathrm{CCA}$ should also be considered, and these patients should be enrolled in clinical (basket) trials.

To perform LT on a patient with advanced-stage cHCC/CCA remains the most controversial treatment decision in our interdisciplinary approach. LT is an established treatment option for early-stage HCC patients, but it does not represent standard of care for patients with intrahepatic CCA or CHCC/CCA. Survival rates following LT are lower among patients with cHCC/CCA than among those with HCC or CCA, and a larger meta-analysis showed no survival benefit from LT over surgical resection in cHCC/CCA patients [2]. However, recent data have suggested a benefit from LT over surgical resection for smaller cHCC/CCAs and intrahepatic CCAs $(<5 \mathrm{~cm})$ in the setting of cirrhosis, and a smaller series of patients with advanced-stage CCA who showed an excellent tumor response to systemic chemotherapy, and were subsequently undergoing transplantation with extended allocation organs that would otherwise have been discarded, revealed a promising 5-year survival rate of $83.3 \%$ [8].

In our case, we were facing a very young patient with an excellent response to chemotherapy but without surgical resectability, no distant metastases, and increasing side effects of systemic treatment limiting its continuation. On the other hand, we still observed a large hepatic tumor manifestation $(>5 \mathrm{~cm}$ ) as well as suspicious hepatic lymph nodes, both representing negative prognostic factors for LT. Nevertheless, we finally decided on performing LT following intraoperative confirmation of node-negative disease, using an extended criteria allocation offer that had been rejected by several transplantation centers, was not suitable for any other LT candidate at our own center, and would otherwise have been discarded. Importantly, the individual decision for LT, which necessitated permanent postoperative immunosuppression, did not limit any further palliative treatment options such as systemic chemotherapy or NGS-based targeted therapies.

When we observed pulmonary tumor recurrence after LT and conventional systemic chemotherapy was no longer applicable due to severe systemic side effects, we decided on an NGS-based diagnostic approach to unravel the tumor's individual genetic landscape and to identify potentially druggable targets. We identified a COLGB1-MET fusion as the most prominent genetic aberration and successfully initiated a targeted therapy with the MET inhibitor crizotinib, which sufficiently controlled tumor growth for almost 1 year. The genetic landscape of cHCC/CCA has only recently come into focus. The most frequently observed genetic mutations in CHCC/CCA are alterations in TERT, TP53, cell cycle genes such as CCND1 or CDKN2A, MET, KRAS, PTEN, and Wnt pathway genes, whereas alterations in genes typically mutated in intrahepatic CCA, such as IDH1, IDH2, and BAP1, are less common [9]. However, these mutations occur very heterogeneously, and no NGS-/mutation-based therapy has been approved for the treatment of cHCC/CCA to date. Interestingly, while we observed good tumor control of almost all pulmonary metastases under treatment with crizotinib, a single metastasis progressed after 7 months of treatment, implying that mutation-based targeted therapy might also be a driver of further genetic alterations. Again, we initiated NGS-based treatment with lenvatinib to target the high-level FGFR3 amplification we found in this specific

\section{Karger'=}




\section{Case Reports in Gastroenterology}

\begin{tabular}{l|l}
\hline Case Rep Gastroenterol 2020;14:658-667 \\
\hline DOI: 10.1159/000511034 & $\begin{array}{l}\text { ○ 2020 The Author(s). Published by S. Karger AG, Basel } \\
\text { www.karger.com/crg }\end{array}$ \\
\hline
\end{tabular}

Loosen et al.: Interdisciplinary Therapeutic Approach to Advanced-Stage cHCC/CCA

metastasis. Although the tumor only partially responded, the NGS-based therapy resulted in sufficient tumor control for over 1 year in a patient that was unable to continue standard chemotherapy and would otherwise have been assigned to active symptom control.

Given its wide heterogeneity in terms of tumor genetics and clinical appearance, the diagnosis of cHCC/CCA has remained challenging, and misdiagnoses (e.g., as HCC or CCA) are commonly observed. Although specific patterns of contrast agent enhancement have been described for $\mathrm{cHCC} / \mathrm{CCA}$ [10], there is no imaging technique allowing a definite noninvasive diagnosis. Thus, early tumor biopsy is recommended if cHCC/CCA is suspected. Nevertheless, a guided biopsy can only reveal information on a small fracture of the whole tumor and might thus not always be fully representative. In our case, the guided biopsy in combination with highly increased levels of AFP, CA19-9, and carcinoembryonic antigen led to the diagnosis of cHCC/CCA. However, our histological analysis of the explanted liver (after local ablative and chemotherapy) did not reveal any viable HCC component, while the biopsy from the bone metastases in the final disease stage showed AFP-positive tumor cells, suggesting an initial treatment response and a dormant HCC clone leading to metastasis during the further clinical course. This scenario is further supported by the decreasing AFP levels after TACE suggesting a treatment response in HCC patients. These findings highlight the diagnostic challenges regarding cHCC/CCA tumors and argue for standardized diagnostic algorithms to allow a definite diagnosis of this heterogeneous tumor entity.

In summary, this case report presents an individual interdisciplinary therapeutic approach for a patient with advanced-stage unresectable cHCC/CCA who clearly outperformed historical periods of survival with long-term survival beyond 5 years. Given the low incidence rate, the lack of specific therapeutic options, and the high heterogeneity of CHCC/CCA, we suggest that these patients should be enrolled in large registries or molecular-driven basket trials whenever possible.

\section{Acknowledgements}

Whole-genome and RNA sequencing was performed within the MASTER program supported by the Molecular Diagnostics Program of NCT Heidelberg and the German Cancer Consortium. The authors thank the NCT/DKFZ Sample Processing Laboratory, the DKFZ Genomics and Proteomics Core Facility, and the DKFZ Omics IT and Data Management Core Facility for technical support.

\section{Statement of Ethics}

The treatment of the patient was conducted ethically in accordance with the World Medical Association Declaration of Helsinki. Written informed consent to publish the case including images was obtained from the patient's wife.

\section{Conflict of Interest Statement}

The authors have no conflicts of interest to declare.

\section{Karger'}




\section{Case Reports in Gastroenterology}

\section{Funding Sources}

There was no direct funding involved in this case report. Work in the laboratory of T.L. was funded by the European Research Council under the European Union's Horizon 2020 Research and Innovation Program through the ERC Consolidator Grant PhaseControl (No. 771083). The laboratory of T.L. was supported by the German Cancer Aid (Deutsche Krebshilfe 110043 and Mildred-Scheel Professorship) and the German Research Foundation (SFBTRR57/P06 and LU 1360/3-1).

\section{Author Contributions}

T.L., S.H.L., M.S., T.H.W., S.K., J.S., F.T., C.T., C.R., and U.P.N. were involved in the direct medical treatment of the patient. N.T.G. and T.Lo. performed the histological analysis; S.U., A.S., H.G., and S.F. were involved in the NGS-based analyses; S.H.L. generated the figures; and S.H.L. and T.L. drafted the manuscript. All authors approved the final version of the manuscript.

\section{References}

1 Wang J, Li E, Yang H, Wu J, Lu HC, Yi C, et al. Combined hepatocellular-cholangiocarcinoma: a population level analysis of incidence and mortality trends. World J Surg Oncol. 2019 Feb;17(1):43.

2 Li DB, Si XY, Wang SJ, Zhou YM. Long-term outcomes of combined hepatocellular-cholangiocarcinoma after hepatectomy or liver transplantation: a systematic review and meta-analysis. Hepatobiliary Pancreat Dis Int. 2019 Feb;18(1):12-8.

3 Kobayashi S, Terashima T, Shiba S, Yoshida Y, Yamada I, Iwadou S, et al. Multicenter retrospective analysis of systemic chemotherapy for unresectable combined hepatocellular and cholangiocarcinoma. Cancer Sci. 2018 Aug;109(8):2549-57.

4 Gruenberger B, Schueller J, Heubrandtner U, Wrba F, Tamandl D, Kaczirek K, et al. Cetuximab, gemcitabine, and oxaliplatin in patients with unresectable advanced or metastatic biliary tract cancer: a phase 2 study. Lancet Oncol. 2010 Dec;11(12):1142-8.

5 Bender S, Gronych J, Warnatz HJ, Hutter B, Gröbner S, Ryzhova M, et al.; International Cancer Genome Consortium PedBrain Tumor Project. Recurrent MET fusion genes represent a drug target in pediatric glioblastoma. Nat Med. 2016 Nov;22(11):1314-20.

6 Kim JH, Yoon HK, Ko GY, Gwon DI, Jang CS, Song HY, et al. Nonresectable combined hepatocellular carcinoma and cholangiocarcinoma: analysis of the response and prognostic factors after transcatheter arterial chemoembolization. Radiology. 2010 Apr;255(1):270-7.

7 Na SK, Choi GH, Lee HC, Shin YM, An J, Lee D, et al. The effectiveness of transarterial chemoembolization in recurrent hepatocellular-cholangiocarcinoma after resection. PLoS One. 2018 Jun;13(6):e0198138.

8 Lunsford KE, Javle M, Heyne K, Shroff RT, Abdel-Wahab R, Gupta N, et al.; Methodist-MD Anderson Joint Cholangiocarcinoma Collaborative Committee (MMAJCCC). Liver transplantation for locally advanced intrahepatic cholangiocarcinoma treated with neoadjuvant therapy: a prospective case-series. Lancet Gastroenterol Hepatol. 2018 May;3(5):337-48.

9 Joseph NM, Tsokos CG, Umetsu SE, Shain AH, Kelley RK, Onodera C, et al. Genomic profiling of combined hepatocellular-cholangiocarcinoma reveals similar genetics to hepatocellular carcinoma. J Pathol. 2019 Jun;248(2):164-78.

10 Sanada Y, Shiozaki S, Aoki H, Takakura N, Yoshida K, Yamaguchi Y. A clinical study of 11 cases of combined hepatocellular-cholangiocarcinoma Assessment of enhancement patterns on dynamics computed tomography before resection. Hepatol Res. 2005 Jul;32(3):185-95. 


\section{Case Reports in Gastroenterology}
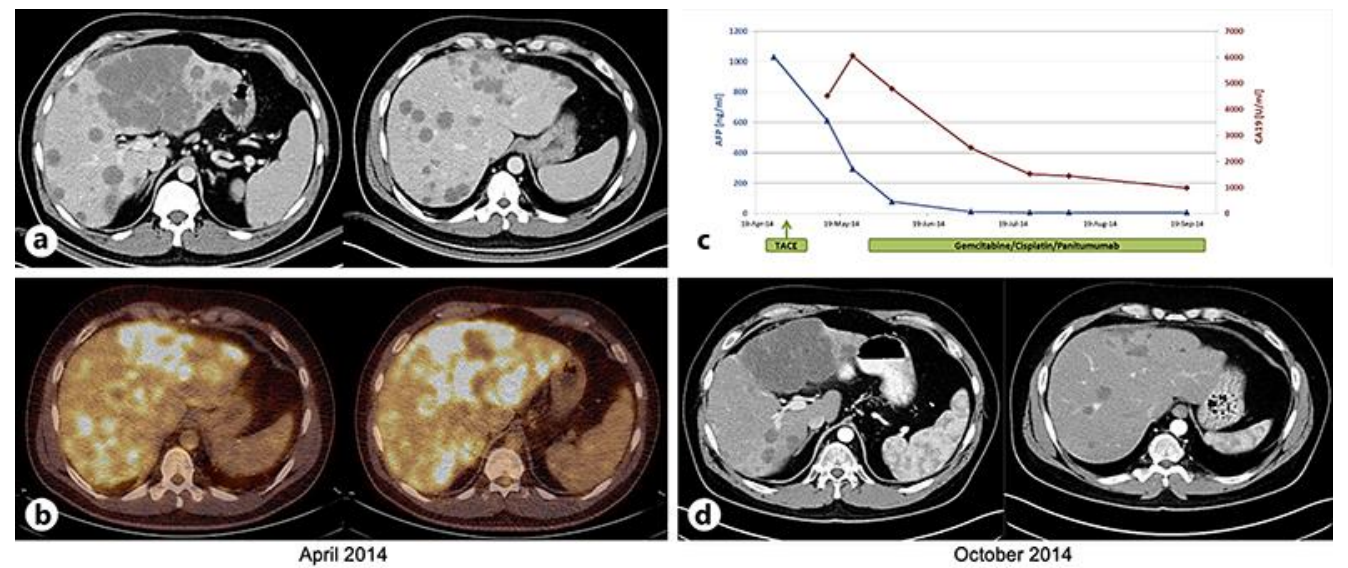

Fig. 1. CT and PET scans of hepatic tumor manifestation and course of tumor markers following initial treatment. a Abdominal CT scan revealing an extensive hepatic tumor manifestation with multiple hypodense liver lesions in all liver segments with a maximum tumor diameter of $12.9 \mathrm{~cm}$. b $18 \mathrm{~F}$-FDG-PET/CT scan confirming the presence of liver-limited disease with multiple intrahepatic sites of FDG uptake but no sign of hypermetabolism in the suspicious hepatic lymph nodes. c TACE therapy resulted in a divergent tumor marker response, with decreasing serum AFP levels but increasing CA19-9 levels. Additional systemic therapy with gemcitabine, cisplatin, and panitumumab resulted in a significant decrease in both AFP and CA19-9 serum levels. $\mathbf{d}$ The combination of TACE and systemic therapy resulted in a significantly reduced hepatic tumor manifestation on abdominal CT scans 6 months after diagnosis of cHCC/CCA. TACE, transarterial chemoembolization; AFP, alpha-fetoprotein; cHCC/CCA, combined hepatocellular-cholangiocarcinoma. 

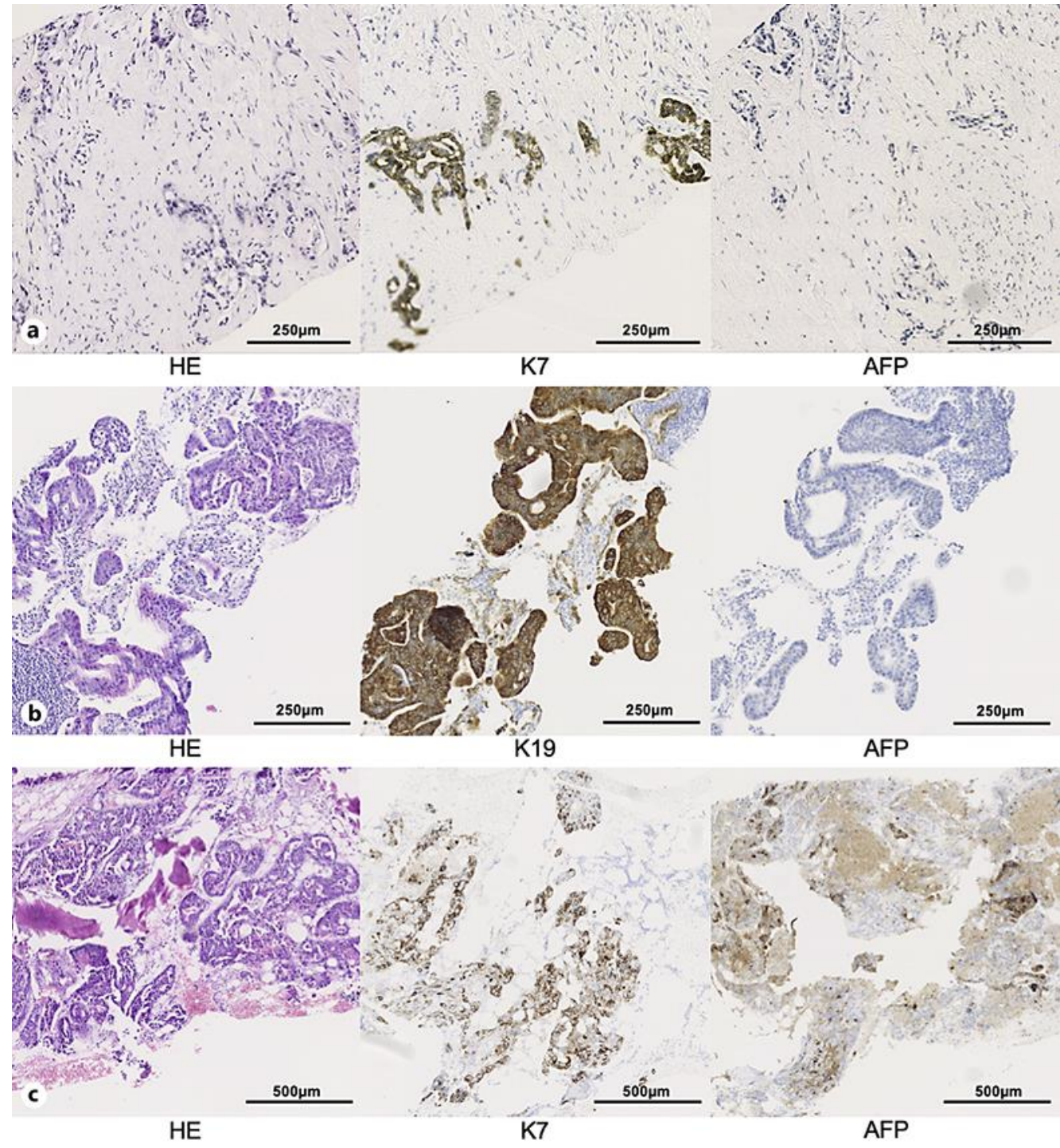

Fig. 2. Histology of the liver tumor, pulmonary metastases, and bone metastases. a CT-guided liver biopsy revealed a poorly differentiated K7-positive but AFP-negative adenocarcinoma, which was morphologically most likely consistent with a cholangiocarcinoma. b CT-guided biopsy of the pulmonary lesions confirmed K19-positive but AFP-negative metastases. c Immunohistochemistry of lumbar vertebral body 3 metastases showed a partially necrotic K7-positive adenocarcinoma now also being positive for AFP. K7/19, keratin 7/19; AFP, alpha-fetoprotein. 